McGill Journal of Education

Revue des sciences de l'éducation de McGill

\title{
ALBERO B., SIMONIAN S., ENEAU J. (Dir). Des humains et des machines. Hommage aux travaux d'une exploratrice. 2019
}

\section{Bruno Garnier}

Volume 54, Number 3, Fall 2019

URI: https://id.erudit.org/iderudit/1069777ar

DOI: https://doi.org/10.7202/1069777ar

See table of contents

Publisher(s)

Faculty of Education, McGill University

ISSN

1916-0666 (digital)

Explore this journal

Cite this review

Garnier, B. (2019). Review of [ALBERO B., SIMONIAN S., ENEAU J. (Dir). Des humains et des machines. Hommage aux travaux d'une exploratrice. 2019]. McGill Journal of Education / Revue des sciences de l'éducation de McGill, 54(3), 683-685. https://doi.org/10.7202/1069777ar 
ALBERO B., SIMONIAN S., ENEAU J. (Dir). Des humains et des machines. Hommage aux travaux d'une exploratrice. Dijon : Éditions Raison et Passions, 2019.

L'ouvrage dirigé par Brigitte Albero, Stéphane Simonian et Jérôme Éneau n'est pas seulement l'hommage annoncé à Monique Linard, professeure émérite de sciences de l'éducation et de la formation à l'Université Paris-Ouest-Nanterre, ancienne directrice d'un laboratoire de sciences humaines et sociales consacré à la formation et aux technologies de l'information et de la communication. Cet ouvrage est aussi un état des lieux de 597 pages composé par quarante-deux contributions de chercheurs francophones de plusieurs disciplines, concernant l'action humaine médiée par les technologies, en particulier la formation et les apprentissages. Pour Brigitte Albero, les travaux de Monique Linard, qui ont couvert quatre vagues technologiques durant les cinq dernières décennies - image, film, vidéo, informatique -, ont ouvert une voie qui consiste à placer au centre de l'action éducative la médiation humaine en tant que processus-clé à tous les âges de la vie. Elle met en évidence une proposition fondatrice de Monique Linard qui consiste à placer de manière centrale l'activité humaine dans toute étude des technologies, afin d'éviter de se laisser entraîner dans la course folle des innovations, au point de perdre la capacité de réfléchir aux missions éducatives premières ou de subordonner l'action éducative aux demandes d'efficacité immédiate.

Au fil de ses sept parties, l'ouvrage semble suivre une direction en quatre moments, bien que ceux-ci ne soient pas explicités. Il s'ancre premièrement dans la vie et l'œuvre de Monique Linard dans leurs aspects généraux (partie 1 « Hommage à une exploratrice " et partie 2 "Un positionnement épistémologique entre connaissance et action, sujets et artefacts »), pour ensuite opérer une focalisation sur le modèle de formation Hélices, qui fait l'objet de plusieurs analyses dans la partie 3 ("Hélices : un modèle de l'activité humaine »). Un troisième moment réunit des approches critiques ou comparatistes (partie 4 «Entre recherche et intervention : des concepts intermédiaires "; partie 5 "Mythes et limites de la vidéoscopie "; partie 6 "Préoccupations praxéologiques en éducation et en formation »). Enfin, un quatrième et dernier moment propose une ouverture 
prospective des travaux de Monique Linard au regard des enjeux des temps actuels et futurs (partie 7 " Perspectives de la recherche " et "Conclusion ").

Si l'ensemble de l'ouvrage tient sa cohérence de son sujet ( les travaux d'une chercheure reconnue ) et d'un objet thématique ( sa manière de valoriser la dimension humaine dans les technologies éducatives), les changements fréquents de points de vue et la brièveté des articles (souvent moins de dix pages) donnent une impression kaléidoscopique qui correspond au souci d'exhaustivité. Dans cet ensemble d'une grande richesse mais un peu touffu, on trouve de véritables perles qu'il n'est pas possible ici de commenter toutes, en relation avec les positions pionnières de Monique Linard. Parmi elles, l'article de Catherine Roby, "Penser le rapport entre science et société », évoque la constitution historique du lien longtemps peu questionné entre science, technique et progrès, et il montre comment, au $\mathrm{XX}^{\mathrm{e}}$ siècle, la technique fut reléguée hors des sphères nobles de la culture, avant le renversement de perspective opéré par la sociologie des sciences dans les années soixante-dix qui se mit à penser les sciences et les techniques dans leurs dimensions politiques et économiques, sociales et éthiques. Jacques Audran revisite cette histoire en mettant d'abord l'accent sur la rupture de la première moitié du XX $\mathrm{XX}^{\mathrm{e}}$ siècle (avec les crises économiques, les guerres, les génocides, la bombe atomique) qui a durablement assombri l'image d'une technique présentée comme inhumaine, puis sur le changement de perspective qui a permis de passer de la technique aux technologies, qui sont « la part humaine de ce qui est technique » (p. 92-93). Il faudrait également saluer le texte de Teresa Yurén sur les enjeux éthiques de la relation entre formation et technologies et aussi la mise en perspective historique proposée par Georges-Louis Baron. À ces éclairages spéculatifs, l'ouvrage ne pouvait manquer de proposer une articulation dynamique avec les dispositifs et plus généralement la praxis des techniques éducatives, en s'inspirant de la démarche de l'« exploratrice " Monique Linard. Et ce pari est tenu, notamment autour du modèle Hélices, imaginé par Monique Linard à partir de 1989 pour tenter de surmonter un certain nombre de confusions, notamment celle qui confond les concepts d'information externe et la connaissance subjectivée par le sujet dans des situations déterminées. Ce modèle, qui comprend l'axe diachronique du pilotage de l'action et l'axe synchronique lié à la mobilisation hiérarchique des dispositifs mobilisés, trace un pont entre l'engagement des acteurs de la formation et l'appropriation des techniques (André Zeitler). Entre recherche d'intelligibilité et opérationnalisation pratique des interventions en éducation et formation, une série d'articles de la quatrième partie développe des concepts intermédiaires, tels que dispositif, médiation ou médiatisation, ingénierie, agentivité et activité (notamment Pascal Marquet). On lira avec intérêt les limites signalées par Monique Linard elle-même par certaines techniques pédagogiques, telles que la vidéoscopie, notamment dans la formation des professionnels de l'éducation (Jérôme Guérin, Serge Leblanc) et de la santé (Catherine Archieri, Joris Thievenaz), mais on s'attardera surtout sur les perspectives ouvertes dans la septième partie et dans la conclusion, à 
propos des collectifs pour apprendre (Jean Heutte) et les conditions d'émergence de nouveaux systèmes d'interaction entre l'humain et la machine (Catherine Garbay). Enfin, il faut souligner la présence d'une bibliographie globale pour tous les articles et d'un glossaire qui a présidé à l'harmonisation des notions et des concepts de tous les textes. L'ouvrage présente enfin la publication de trois textes inédits de Monique Linard particulièrement contemporains.

Au total, cet ouvrage foisonnant pose de nombreuses questions qui nous interpellent, à l'heure où l'intelligence artificielle gagne des secteurs de l'activité humaine de plus en plus nombreux. Or les machines pour apprendre « ne doivent pas juguler l'imagination des humains, leur besoin de liberté et de dialogue au risque sinon de transformer nos sociétés en territoires proprement invivables " (Françoise Thibault, p. 463), et nous laisserons le dernier mot à Monique Linard elle-même (1987, p. 27), qui préconise la prise en compte « fondamentalement incarnée et multidimensionnelle de l'activité humaine ».

BRUNO GARNIER Université de Corse

\section{RÉFÉRENCE}

Linard, Monique (1987). Nouvelles technologies et représentation cognitive : vers une pédagogie de l'incertitude. Actes du colloque de l'AECSE Culture technique et formation, Nancy : PUN. 